\title{
Estudio sobre el sentido de pertenencia y percepción de los alumnos de la Licenciatura en Administración de la Universidad Autónoma Metropolitana Unidad Azcapotzalco
} A study on the sense of belonging and students perception on the Management Bachelor at the UAM, Azcapotzalco unit.

Recibido: 25 de febrero de 2016

Evaluado: 9 de junio de 2016 Aceptado: 30 de junio de 2016

\author{
César Medina Salgado (México) \\ Doctor en Estudios Organizacionales \\ Universidad Autónoma Metropolitana \\ medina.cesar24@gmail.com
}

\section{Resumen}

El documento explora los sentimientos percibidos por las alumnas y alumnos de la Licenciatura en Administración de la Universidad Autónoma Metropolitana Unidad Azcapotzalco. ¿Por qué indagar sobre este fenómeno? La pesquisa se orienta hacia el descubrimiento de los sentimientos de identificación y arraigo experimentados por los estudiantes en su estancia académica en la UAM Azcapotzalco. Porque su futuro profesional dependerá en gran medida de los nexos emocionales establecidos con sus compañeros.

Palabras clave: sentimientos, administración, estudios de administración, sentido de pertenencia.

\section{Summary}

This paper explores the feelings perceived by students on the Management Bachelor at the UAM, AZCAPOTZALCO unit. Why investigate this phenomenon? This inquiry is oriented towards the discovery and identification of feelings and sense of belonging that is experienced by students during their academic stay at the UAM Azcapotzalco. Their professional future will largely depend on the emotional links established with their peers.

Keywords: feelings, management, management studies, sense of belonging. 



\section{Introducción}

El presente artículo forma parte de una investigación desarrollada en la Universidad Autónoma Metropolitana Unidad Azcapotzalco (UAM Azcapotzalco) adscrita al Proyecto Ética y Administración. En él se exploran los sentimientos percibidos por las alumnas y los alumnos de la Licenciatura en Administración de la UAM Azcapotzalco. ¿Por qué indagar sobre este fenómeno? La pesquisa se orienta hacia el descubrimiento de los sentimientos de identificación y arraigo experimentados por los estudiantes en su estancia académica en la UAM Azcapotzalco.

La determinación de los sentimientos de los alumnos permitirá conocer cuáles son los posibles efectos provocados en ellos por el currículum oculto y nulo (aprendizajes adquiridos por las personas sin haber sido planteados dentro del plan de estudios como un objetivo pedagógico expreso) latentes en la UAM Azcapotzalco y, en particular, cómo los viven los alumnos de la Licenciatura en Administración. Simultáneamente la investigación podría arrojar rudimentos incipientes sobre el porqué de la visión utilitarista de algunos estudiantes con respecto a los conocimientos propios de su disciplina: la administración. En otros términos, tan solo estudian por los sueldos futuros y no por vocación. Esta visión en la mayoría de los casos es la antípoda de la acuñada por la tradición académica universitaria.

$\mathrm{El}$ asunto de arranque se basa en una información incierta y desconcertante sobre el quehacer educativo en la UAM Azcapotzalco. Existe un rumor propagado por algunos egresados en conversaciones informales donde manifestaron haber sentido un dejo de rechazo al solicitar sus primeros empleos, lo cual se podría considerar natural quizá por no ajustarse al perfil del puesto solicitado por la entidad contratante. Sin embargo, parece existir un fenómeno ulterior cuando descubren un elemento común entre el reclutador y ellos: ambos candidatos son egresados de la Licenciatura en Administración de la UAM Azcapotzalco. Es decir, desde el punto de vista de los egresados su rumor pareciera encerrar una sentencia grave: ${ }$ Un alumno UAM no contrata a otro alumno UAM!

Por la situación anterior se inició un rastreo en torno a esta presunción exteriorizada por los egresados. De este "mito" nacen un conjunto de interrogantes: ¿por qué ocurre este fenómeno?, ¿`en realidad es cierto?, ¿la segmentación del plan de estudios influye en la integración de los alumnos de la Licenciatura en Administración?, ¿el "currículum oculto" y el nulo están provocando problemas heterogéneos de identidad o pertenencia en los alumnos de la UAM Azcapotzalco? Bajo este telón de fondo se justifica el objetivo de investigación y la discusión escolástica en torno al por qué realizar un estudio sobre la pertenencia de los alumnos de la Licenciatura en Administración de la UAM Azcapotzalco.

Para tratar de avanzar alguna suerte de respuesta, es conveniente recuperar las afirmaciones de Pierre Bourdieu sobre la sociología, que debe objetivar al mundo práctico tomando en cuenta las vivencias de los individuos en lo más inmediato de su entorno, de tal forma que esta ciencia no se aleje de la realidad cotidiana de los seres humanos (Bordieu, 1990; La Recherche, 2000).

En el sentido asignado por Bordieu a la sociología, se esquematiza la dialéctica entre lo personal e íntimo (los habitus del individuo), con lo macrosocial (campos y espacios sociales). De esta forma, la teoría de Bourdieu (1990) posibilita la ampliación de los objetos de estudio (Guerra Manzo, 
2010; Capdeville, 2011) y analiza eventos antes impensables, como los sentimientos.

Los sentimientos emergen de las relaciones sociales donde el individuo se encuentra implicado. En consecuencia, se puede afirmar que los sentimientos son una construcción social, porque las personas sienten de manera imperceptible apego a ciertas formas socialmente establecidas e inducidas. Así, se constituyen las convenciones basadas en los sentimientos (Hochshild, 2008, p. 129; Javeau, 2010); es decir, las personas siguen un principio organizador socialmente impuesto (Hormigos y Oda, 2014). Quizá mediante este principio materializan un conjunto de arquetipos o estereotipos introyectados en sus mentes por la sociedad.

De manera concomitante con el proceso descrito en el párrafo anterior se desencadena

la interacción intercultural, o sea, la interacción entre sujetos diferentes, no solo en su individualidad, sino en aquello que los distingue como miembros de grupos sociales y culturales distintos, lo que conduce a que de la interacción misma puedan desprenderse los significados subyacentes desplegados por los sujetos, así como las posiciones sociales desde donde actúan en el mundo. (Pech, Rizo y Romeu, 2009, pp. 35-36)

Por otra parte, es pertinente resaltar la génesis de la percepción humana, ya que las personas reciben los primeros efectos del medio ambiente a través de sus sentidos (El cerebro, el universo dentro de nosotros: percepción, s/f); Arndt, 2012). En consecuencia, el ser humano cuenta con la capacidad de sentir, porque percibe (siente) el frío, el calor, el hambre, el dolor en alguna parte de su cuerpo. Es decir, conforme avanza la edad puede sentir la hostilidad de su medio, el amor, el abandono, la protección, la envidia, la seguridad, los celos, la vergüenza, la alegría, la tristeza, entre muchas otras formas de emociones traducidas en sentimientos (Otero, 2006). En suma, desde el nacimiento todavía no se actúa, ni se piensa pero ya se siente (Heller, 1999). Por tanto, la capacidad de sentir no es aprendida sino inherente al ser humano, no puede haber pensamiento sin sentimiento (Heller, 1999).

A pesar del origen innato de la capacidad de sentir, también se puede considerar como el resultado de la educación, de la asimilación de normas y del proceso de aprendizaje: los sentimientos son las emociones reales surgidas al estar implicado en algo. Este algo puede ser otro ser humano, un concepto, él mismo, un problema o una situación (Heller, 1999).

Si los sentimientos fuesen solo estados internos o innatos, un determinado hecho afectaría por igual a todas las sociedades y personas en el orbe, y no sucede así. El abandono, la muerte, el nacimiento, la relación con los padres, el rompimiento con la pareja, entre muchos otros, conforman sucesos no vividos de la misma forma en las distintas sociedades (Laneri, 2007). En cada una de ellas, se pueden estudiar las consecuencias emocionales y los sentimientos generados por dichos advenimientos.

Por tanto, la premisa sustentada en los objetos como detonantes de los sentimientos, a pesar de ser desconocidos o difusos en muchas situaciones pueden ser dirigidos, regulados por costumbres y ritos sociales de la cultura. Dentro de este correlato propuesto por Heller, cabe preguntarse: ¿qué son los sentimientos? En la siguiente sección, se darán algunos elementos de respuesta a este cuestionamiento.

Por último, en esta introducción es pertinente exponer la taxonomía del documento consistente en tres subacápites: 1 . 
Un breve escrutinio sobre los sentimientos. 2. El microinteraccionismo simbólico como andamiaje teórico. 3. Análisis de resultados. Asimismo se dirige a todas las personas interesadas en el tema.

\section{Un breve escrutinio sobre los sentimientos}

Para iniciar esta sección, es pertinente preguntar ¿qué es un sentimiento? A fin de responder al interrogante anterior, se recurrió al Diccionario de la Real Academia Espańola, que define el sentimiento como: 1) "Hecho o efecto de sentir o sentirse". 2) "Estado afectivo del ánimo".

En la misma fuente, se especifica la palabra sentir (del lat. Sentìre) bajo las siguientes acepciones: 1) Experimentar sensaciones producidas por causas externas o internas. 2) tr. Oír o percibir con el sentido del oído. 3) tr. Experimentar una impresión, placer o dolor corporal. 4) tr. Experimentar una impresión, placer o dolor espiritual. 5) tr. Lamentar, tener por doloroso y malo algo. 6) tr. Juzgar, opinar, formar parecer o dictamen. 7) prnl. Dicho de una persona: Formar queja de algo. 8) prnl. Padecer un dolor o principio de un daño en parte determinada del cuerpo. 9) prnl. Hallarse o estar de determinada manera. 10) prnl. Considerarse, reconocerse.

De esta forma los sentimientos pueden ubicarse como uno de los caminos más usuales donde la afectividad se da a conocer, y este concepto surge de la expresión derivada del latín sentire que significa pensar, opinar o darse cuenta de algo. Descartes ya en el siglo XVII ofrece una aproximación sobre su definición y la asocia a estados interiores pasivos muy difíciles de concretar a nivel verbal. En la Ilustración francesa, con Rousseau, en su obra Julia, o la nueva Eloisa, aparecen alusiones a los sentimientos esenciales. Aunque es a lo largo del siglo XIX, con el Romanticismo, cuando se vuelve a la exaltación de los sentimientos imprescindibles para la creación artística del momento.

El sentimiento es la experiencia más destacada dentro de la vida afectiva de las personas, definiendo este término como "estado subjetivo difuso, que tiene siempre una tonalidad positiva o negativa" Rojas (1993, p. 59), citado por Álvarez (2002, p. 3), lo considera como un estado subjetivo en cuanto que la experiencia básica del sentimiento reside en el yo; difuso porque su percepción no es muy clara ni muy específica. En los sentimientos la neutralidad no existe, ellos oscilan entre lo agradable o desagradable, y desde lo positivo a lo negativo.

En el Diccionario filosófico-pedagógico (1997, p. 511), se define este aspecto de la afectividad como el "estado de ánimo o disposición afectiva, de agrado o desagrado, hacia personas, objetos, sucesos, opiniones". Hace referencia, además, a distintos enfoques. Hay quienes consideran que sentimiento es igual a emoción. Otros opinan que los sentimientos van separados de las sensaciones (Álvarez, 2002, p. 3). Quizá el mayor defensor de esta última perspectiva sea el filósofo alemán Max Scheler (1992), quien hace una clasificación de los sentimientos en cuatro categorías: 1 . Sensoriales (localizados en el cuerpo -dolor). 2. Vitales (más generales y sin localización concretasalud). 3. Anímicos o psíquicos (desligados del cuerpo-tristeza). 4. Espirituales (trascendentes-paz) (Gabás, 2002; Escudero, 2007).

En opinión de Gaytán (2011), la taxonomía de Scheler va desde lo inmotivado endógeno a lo motivado reactivo y desde una raíz somática hasta otra psicológica y espiritual. De esta manera los sentimientos 
de los estratos más profundos se corresponden con situaciones afectivas primarias ligadas a la corporalidad, reflejo de alteraciones biológicas. Por contra, las capas más superficiales se corresponden a sentimientos más elaborados, relacionados con las circunstancias de la persona.

Los "sentimientos", como el amor, el odio, la angustia, entre otros, son claramente distinguibles por sus causas subyacentes más complejas, su duración más prolongada y menor intensidad, si se comparan con las emociones (ver Hentschel [2001] para una explicación bioquímica), aunque a menudo se construyen sobre un vínculo emocional con los objetos específicos generadores del sentimiento y se evocan incluso en ausencia de estos (Consier, 2006, pp. 6-7).

En opinión del psiquiatra Luc Ciompi (2007), no se sabe, o al menos la ciencia no lo sabe con certeza, qué es un sentimiento, cuál es su significado y cómo actúa. Teniendo en cuenta de manera habitual a los sentimientos como inasibles, irracionales y perturbadores, se admite su eliminación en lo posible de cualquier "pensamiento objetivo". De ahí se deduce que cualquier ánimo de comprender al otro o al mundo se presenta como algo tergiversado y exclusivamente cerebral.

Para estudiar los fenómenos gestados por los sentimientos, se recurre aquí a la corriente sociológica conocida como interaccionismo simbólico, bautizada con este nombre en 1938. Ella parte de la importancia de la comunicación en el desarrollo de la sociedad, la personalidad y la cultura. Surgida en la Escuela de Chicago, que trató de responder al predominio de la investigación positivista empírica que destacaba en los Estados Unidos durante el primer tercio del siglo XX, el interaccionismo simbólico tiene sus raíces históricas en el pragmatismo y el conductismo (Rizo, s/f, p. 79).
Por otra parte, cabe destacar el aporte metodológico del sociólogo estadounidense Randall Collins, quien acuñó el concepto de cadenas rituales de interacción. Incluso llevó más lejos este postulado sociológico al señalar las microinteracciones, en conjunto como un constituyente fundamental de la vasta estructura de clases en la sociedad moderna.

La microsociología es la corriente de la sociología que se ocupa de la interacción social a escala reducida. Esta corriente, en la que se insertan propuestas como la Etnometodología, el Construccionismo Social o el Interaccionismo Simbólico, se preocupa por los comportamientos cotidianos y las relaciones y vínculos - preferiblemente cara a cara- entre sujetos. [A fin de] ampliar los puntos de vista en torno a la interacción y la comunicación humana. (Rizo, s/f, p. 1674)

Dentro de este campo de conocimientos sociológicos se recurre al microinteraccionismo individual como una posible herramienta para desentrañar el problema de investigación en torno a la pertenencia y el ejercicio profesional solidario de los alumnos de la Licenciatura en Administración de la UAM Azcapotzalco.

\section{El microinteraccionismo simbólico como andamiaje teórico}

Por las ideas expresadas en la sección anterior, el marco teórico de la presente investigación se construirá a partir de las nociones del sociólogo canadiense Erving Goffman. Principalmente aquellas referentes a la sociología microinteraccionista individualista 
simbólica desarrollada en situaciones, episodios o encuentros estructurados de copresencia física. Es decir, el ámbito analítico donde se despliega la interacción "cara a cara” (esta visión de la comunicación social podría etiquetarse como etológica).

En este sentido, Goffman afirma que la sociedad se construye mediante interacciones simbólicas cotidianas entre los individuos. Cada individuo se considera como un actor presentando un personaje, es decir, un "trabajo de cara" (aquí se utilizarán las categorías imagen personal o trabajo de cara indistintamente) ante el resto de los individuos en el foro constituido por una situación social determinada (ya sea casual o estructurada).

Entonces el objeto de la perspectiva dramatúrgica de Goffman (citado por Herrera y Soriano, 2004, p. 64) es la acción de un actor - o de un equipo de actores - que pretende representar un personaje o una singular rutina ante un público. Por tanto, el actor siempre se presenta frente al público (y la observación sociológica) con los "ropajes" de un personaje en particular (Goffman, 1956, p. 482).

En consecuencia la dramaturgia sí se observa como ritual (Caballero, 1998, p. 127). Crea un sentido de realidad compartida, realidad que no es forzosamente efímera. En la medida en la que el ritual tiene éxito, crea símbolos sociales llenos de fuerza moral. Los participantes salen de un ritual creyendo en los símbolos, al menos durante cierto tiempo. La realidad social no es solo construida, sino también reproducida y mantenida. Cabe decir que los rituales tienen un carácter coercitivo.

Por lo anterior para entender el ritual es necesario contar con algunas descripciones de los elementos participantes en él. El primer componente y la raison d'être del presente estudio es esta: la interacción (es decir, la interacción cara-a-cara). De forma sucinta puede ser definida como la influencia recíproca de los individuos sobre las acciones conexas cuando están en mutua presencia física inmediata.

Otro elemento del ritual es la actuación consistente en toda actividad realizada por un participante dado en una ocasión determinada, que es utilizada para influir sobre cualquiera de los demás participantes. En esta actividad, se incluyen sus gestos, ademanes, tono de voz, mirada, manera de vestir, entre otros elementos. Con respecto a los participantes y sus acciones vistas desde la óptica de un participante en concreto y a partir de su actuación como punto de referencia, son considerados como audiencia, observadores o coparticipantes.

Siguiendo con la taxonomía ritualista se tiene la pauta de acción preestablecida (el guion) desplegada durante una actuación en una o varias ocasiones, por ello, podría ser denominada parte o rutina. Cuando un individuo o actor realiza la misma parte o papel ante la misma audiencia en distintas ocasiones, existe alguna probabilidad para el establecimiento de una relación social. De esta manera (quizá desde una visión funcionalista parsoniana) el rol social se define como la realización de derechos y deberes ligados a un estatus dado, consecuentemente un rol social incluirá una o más partes (Goffman, 1990, pp. 13-14).

Dentro de la situación social descrita previamente resalta un conjunto de particularidades, como la manera de presentar el personaje y la apariencia (gestual o no) de los individuos. Además subyacen en los actores: 1) pensamientos, 2) acciones, 3) sentimientos y 4) emociones. Consecuentemente el actor deberá pasar por estados de autoconciencia de sus emociones. En opinión de Hochschild (2008, p. 136), el actor debe ser capaz de evaluar cuándo 
un sentimiento es inapropiado y hacer el intento de manejarlo. Así, a través de su sensitividad hacia el personaje desempeñado (Hochschild denomina esta mirada interior "actuación profunda"), se logra poner en marcha el mecanismo de interacción con las otras personas con quienes coexiste el actor- de forma repetitiva o eventual.

De los elementos subyacentes en el actor se recupera de los párrafos superiores el inciso marcado con el número tres correspondiente a los sentimientos. Porque los sentimientos aquí se consideran como un mecanismo hermenéutico para entender el comportamiento de los alumnos de la Licenciatura en Administración de la UAM Azcapotzalco. El ejercicio de interpretación podría abarcar los ámbitos de su estancia estudiantil en un primer momento y eventualmente en el futuro el construido una vez egresados de la UAM Azcapotzalco.

\section{Análisis de los resultados Algunos antecedentes}

Para los fines del presente trabajo, la UAM Azcapotzalco se considera como una institución, porque en esos espacios particulares se puede verificar cómo los grupos sociales forman una vida propia que, vista de cerca, se vuelve "significativa, razonable y normal” (Goffman, 1961, pp. 9-18), un universo determinado por construcciones colectivas que los integrantes de una comunidad reconocen y revitalizan en su actuar cotidiano (citado por Mercado y Zaragoza, 2011, p. 161). De igual forma al ser la UAM Azcapotzalco una institución se establecen en su interior un conjunto de mecanismos tendientes al establecimiento del solitudinem (aislamiento) de las relaciones sociales con respecto a su ámbito inmediato exterior. Una especie de asepsia social de las acciones desarrolladas en su interior.

A pesar de este aislamiento, la UAM Azcapotzalco proporciona a la sociedad un conjunto de profesionales abocados a la solución de los problemas nacionales. Esta institución en los últimos cinco años ha formado a 6832 alumnos (1366 en promedio anual) (COPLAN, 2016) mediante sus tres grandes divisiones académicas: Ciencias Básicas e Ingeniería , Ciencias Sociales y Humanidades y Ciencias y Artes para el Diseño. Aquí se analizarán los resultados de las Ciencias Sociales y Humanidades, porque forma parte de ella la Licenciatura en Administración. En la figura 1, se muestran en porcentajes los puestos desempeñados por los egresados de esta división académica. 
Figura No. 1. Egresados de la División de Ciencias Sociales y Humanidades (generaciones 2005-2008).

Actividades Abandonadas

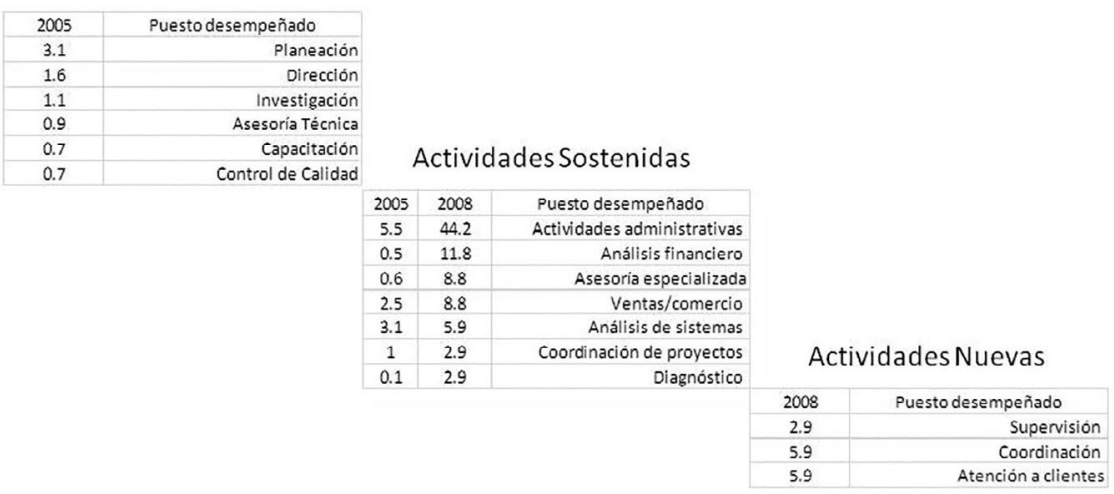

Fuente: Tomado de http://www.egresados.uam.mx/EstSeg20052008.html

En la figura 1, se agruparon en tres secciones las actividades profesionales de los egresados. La primera sección refleja las actividades (abandonadas) desempeñadas por la generación 2005 y no mencionadas por la 2008. La segunda (actividades sostenidas) muestra las actividades comunes a ambas generaciones y en la tercera las actividades (nuevas) desarrolladas por la generación 2008 y no mencionadas por la 2005.

Para la generación 2008, existe un aumento significativo en las actividades profesionales desarrolladas por los egresados. Destacan las actividades administrativas, las de análisis financiero, las de asesoría especializada y las de ventas/comercio. Incursionan en nuevos campos como la supervisión, la coordinación y la atención a clientes. Mientras tanto la generación 2005 deja la planeación, la dirección, la investigación, la asesoría técnica, la capacitación y el control de calidad.

Por otra parte, y en tiempos recientes, la Licenciatura en Administración evidencia un egreso de 904 alumnos de 2011 a 2015, lo cual representa una tasa de egreso anual promedio de $31 \%$ con respecto al total de la división (2908 alumnos en el mismo periodo). De los 904 egresados 542 se han titulado tras lo cual han consolidado una tasa de $60 \%$ en la eficiencia terminal.

En esta sección, se presentan de manera condensada los resultados obtenidos en un sondeo entre los alumnos de la Licenciatura en Administración de la UAM Azcapotzalco. Los testimonios obtenidos constituirán un primer elemento de investigación para entender el comportamiento de los alumnos. Simultáneamente proporcionarán algunos argumentos para comenzar a delimitar el "trabajo de cara" de los alumnos de la Licenciatura en Administración. En forma paralela, se escudriñan (parafraseando a Goffman, 1970) los signos exteriores e interiores de orientación y de compromiso, estados de la mente y el cuerpo generalmente no examinados en relación con la organización social. 
En palabras de Achón et al. (2013 pp. 93-94):

"El ingreso a la Universidad usualmente coincide con el segundo estadio de la adolescencia y, a su vez, constituye la entrada a una Situación Social de Desarrollo que se distingue por nuevas complejidades para el desenvolvimiento de los jóvenes. Este tránsito obliga a los jóvenes a desarrollar una serie de conocimientos, habilidades, destrezas, actitudes y valores que les permitan una adaptación activa a las nuevas exigencias, nuevos tipos de interacciones entre iguales, de pareja y también a las nuevas oportunidades de libertad y de placeres ya mucho más independientes del tutelaje familiar.

De este modo, el desarrollo psicológico de los adolescentes que ingresan en la Universidad deberá reajustarse al aumento del rigor del estudio, la multiplicidad de tareas docentes, de relaciones sociales, etcétera. En esta situación, resulta fundamental que los adolescentes estén preparados para evitar cualquier conducta riesgosa y establecer un equilibrio entre las nuevas exigencias, las alternativas de desarrollo y su bienestar.

Para lograr los ambiciosos objetivos de investigación, la muestra empleada fue cualitativa e intencional, y estuvo conformada por grupos naturales de alumnas y alumnos de la Licenciatura en Administración de la UAM Azcapotzalco inscritos en el segundo trimestre. Ambos grupos estuvieron de acuerdo en participar en la presente investigación.

Los datos se recopilaron en dos momentos diferentes (trimestres 15-P mayo-julio] y
15-O [septiembre-diciembre]) y se estudiaron los resultados obtenidos en dos etapas. En la primera, se compararon por género (primero mujeres y después los hombres) y en la segunda se analizaron los datos recabados en ambos grupos de manera general como si fuesen una muestra única.

El tamaño del primer grupo fue de 31 estudiantes (14 alumnos [45\%] y 17 alumnas [55\%]) con una edad promedio de 22 años para los hombres y de 21 para las mujeres. El segundo grupo estuvo conformado por 38 estudiantes (18 alumnos [ $48 \%$ ] y 20 alumnas [52\%]) con una media de edades de 22 años para ambos géneros. En los dos grupos, la representación de las alumnas fue mayor. Ambos grupos integraron una muestra de 69 estudiantes.

Aquí es pertinente resaltar el propósito de la muestra (Quinn, 2001, pp. 106-121; Luborsky y Rubinstein, 2001, pp. 122-140) como un instrumento cualitativo orientado hacia la obtención de información, cuyo estudio permitirá enriquecer o dilucidar las preguntas de investigación. Sin pretender arribar a través de ella a conclusiones robustas desde el punto de vista estadístico, ni de carácter universalista.

Para realizar el sondeo, se les planteó a las alumnas y los alumnos la siguiente pregunta: ¿cuáles son los sentimientos experimentados por ellas y ellos al estar en la Universidad? Posteriormente se entregó a cada uno una hoja con la imagen mostrada en la figura 2 y se les solicitó marcar de manera libre (sin un orden preestablecido) los sentimientos reconocidos por ellos en ese momento (figura 2). 
Figura No. 2. Versión modificada de la rueda de emociones de Ginebra.

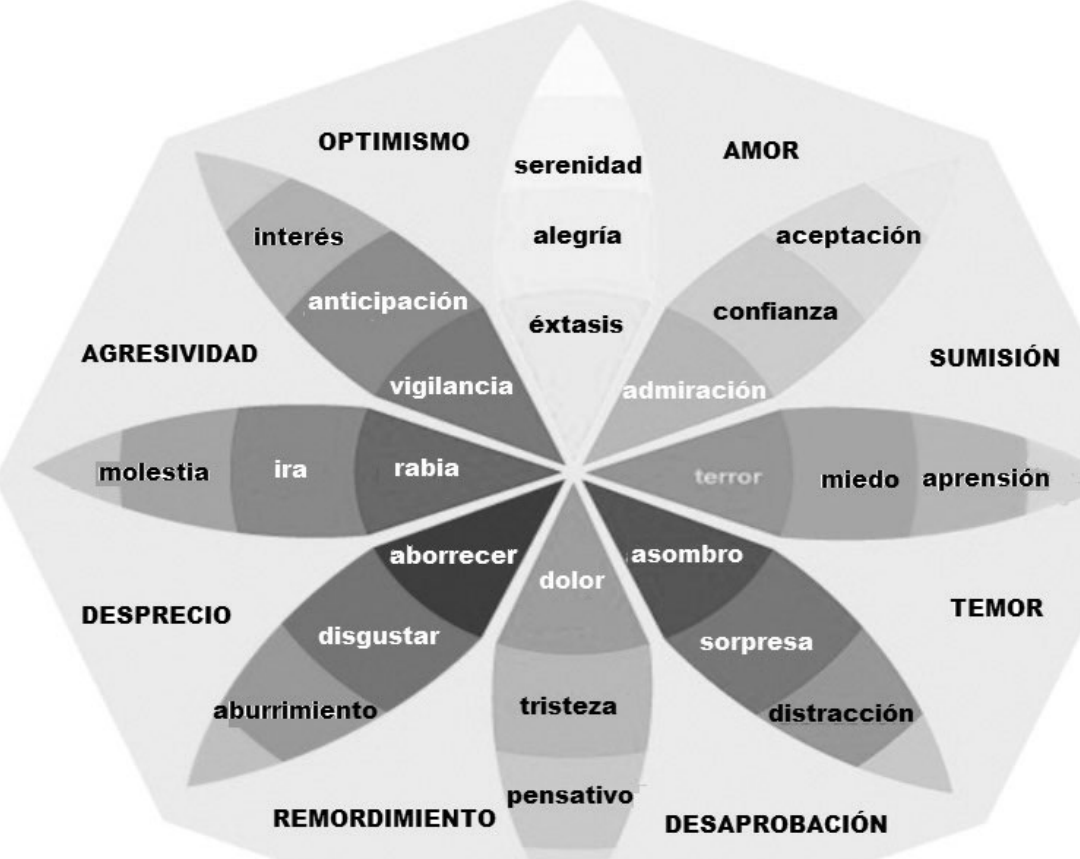

Fuente: Scherer (2005, p. 723).

Como resultado de haber aplicado el instrumento anterior, se puede observar la percepción de un conjunto de sentimientos hacia donde convergen ambos grupos de alumnas y alumnos.

En primera instancia, se analizan los datos obtenidos de los dos grupos de alumnas y en la figura 4 se relacionan los sentimientos señalados por ellas, aunque resaltan algunas diferencias en las frecuencias obtenidas en cada grupo, como se presenta en la figura 5. El coeficiente de correlación entre ambos grupos de alumnas mostró una relación positiva alta $(0.87)$ a partir de los puntajes obtenidos en los sentimientos expresados por cada una de ellas (figura 6).

En ambos grupos de alumnas, el miedo obtuvo la misma ocurrencia. También destacan la distracción y el estar pensativas, fundamentalmente por las diferencias tan amplias manifestadas por ambos grupos. Como un argumento preliminar hacia el entendimiento de estos sentimientos, se presentan sus definiciones en la figura 3. A partir de estas se puede aventurar como conjetura que las alumnas meditan intensamente sobre la posible ocurrencia de sucesos contrarios a sus deseos y apartan su atención de aquello a lo cual deberían estar aplicadas, es decir, a sus estudios. En gran medida, este tipo de miedos están causados por situaciones relacionadas con la autoestima personal y se presenta con mayor incidencia en las mujeres (González-Arratia, Valdez y Serrano, 2015). 
Figura No. 3. Sentimientos que expresan la preocupación de las alumnas.
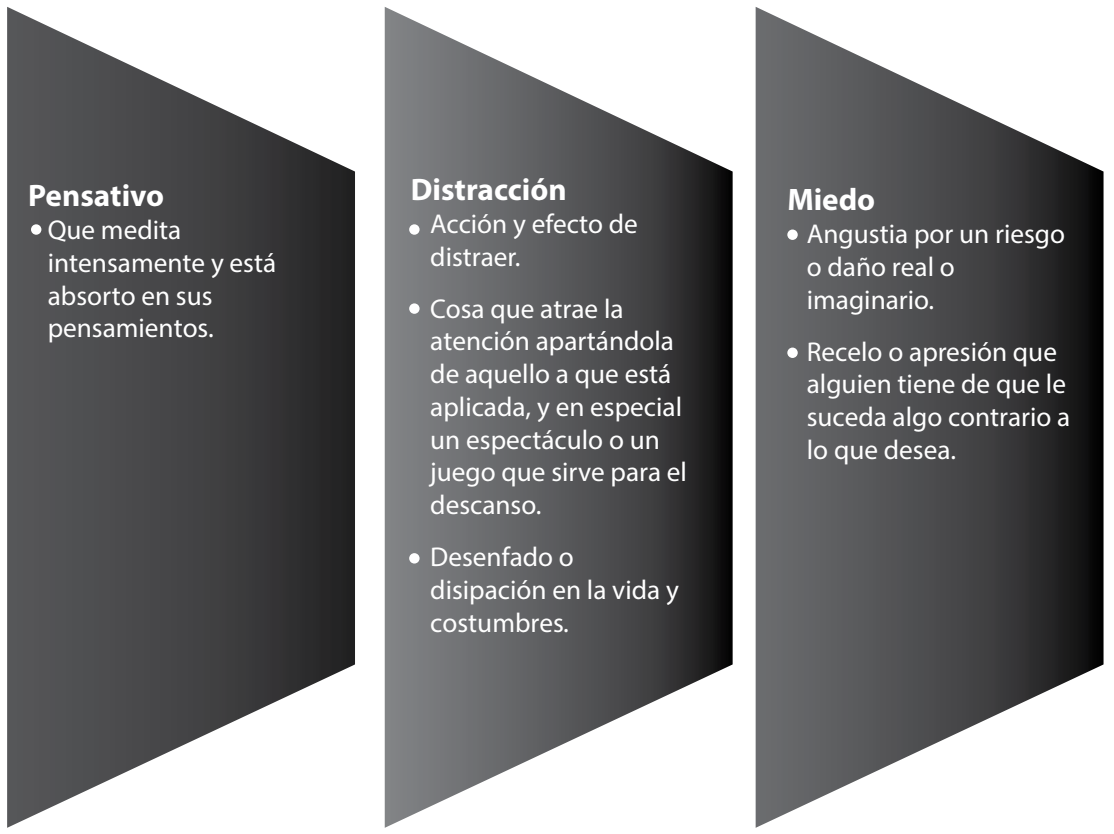

Fuente: Elaboración propia.

Además "el miedo, como reacción negativa o de carácter desagradable de especial relevancia adaptativa y de supervivencia, que se inicia con el procesamiento de estímulos que desencadenan una cascada fisiológica y conductual.

Varios de los aspectos que atemorizan a las personas pueden considerarse determinados biológicamente, entre los cuales la edad es uno de los factores que influyen en la intensidad y el número de miedos, que decrecen con el paso del tiempo hasta alcanzar la edad adulta (Kushnir, Gothelf y Sadeh, 2014; Miloyan, Bulley, Pachana y Byrne, 2014; Pulido y Herrera, 2016). El género es otra variable determinante en la incidencia e intensidad de los miedos (Burnham, Hooper y Ogorchock, 2011). Las diferencias descritas para la variable sexo, hacen que Matesanz (2006) llegue a aconsejar aplicarlos por separado para ambos sexos. Resulta importante destacar la enorme incidencia que tiene el entorno social y cultural sobre el miedo, en que se observa la influencia de los acontecimientos externos en el desarrollo emocional (Roth et al., 2014; Pulido y Herrera, 2016). 
Figura No. 4. Frecuencias absolutas de ambos grupos de alumnas.

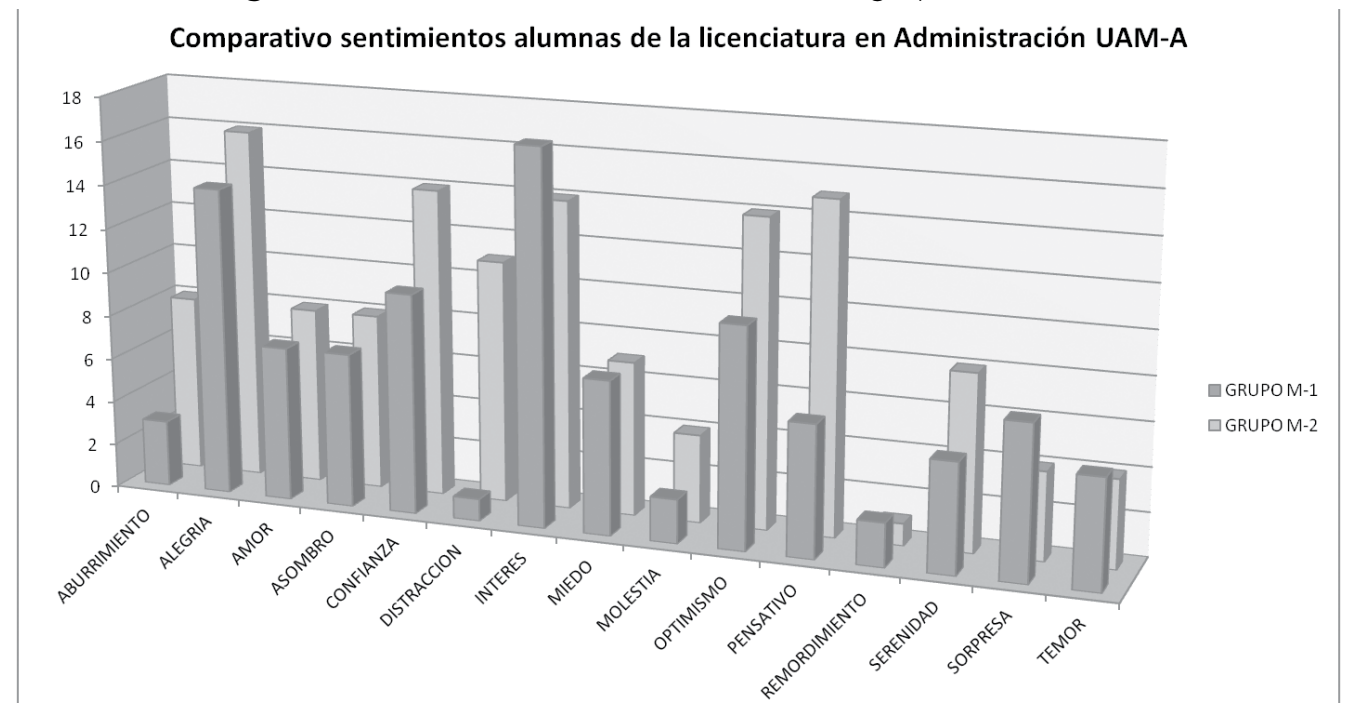

Fuente: Elaboración propia.

Figura No. 5. Oscilación observada en los sentimientos mencionados por ambos grupos de alumnas.

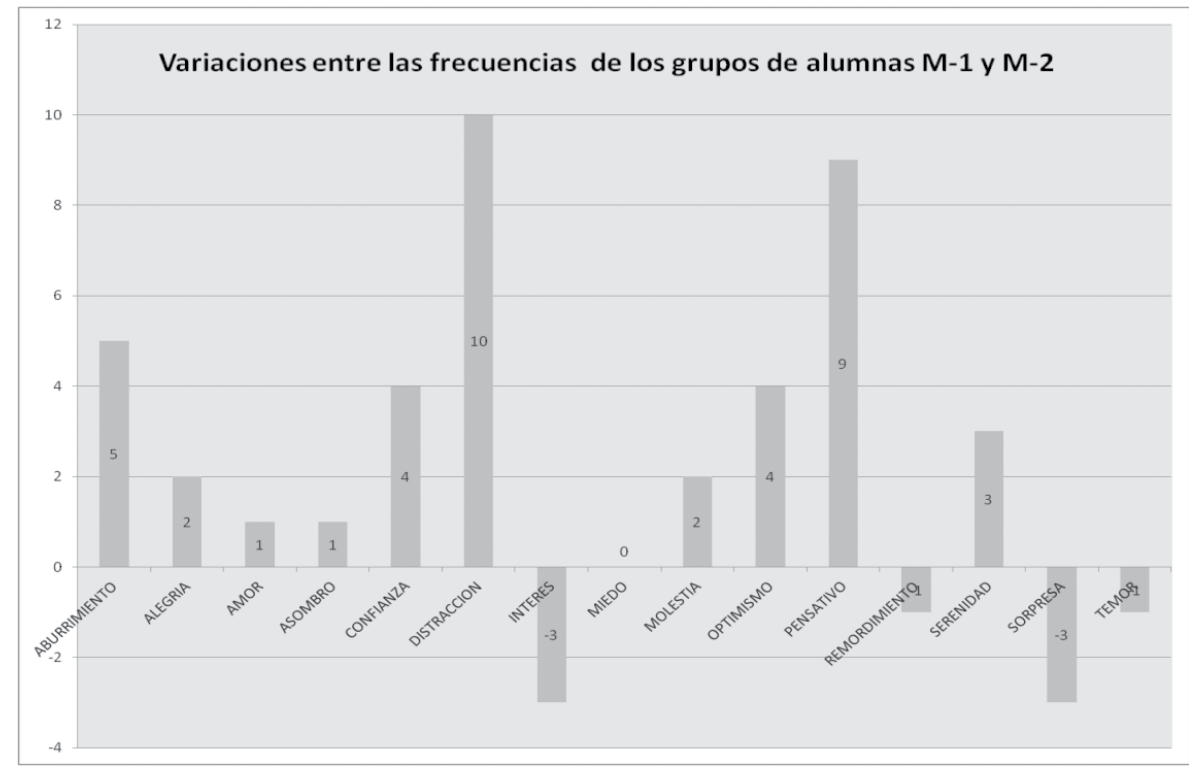

Fuente: Elaboración propia. 
Figura No. 6. Diagrama de dispersión de los sentimientos de ambos grupos de alumnas. Fuente: Elaboración propia.

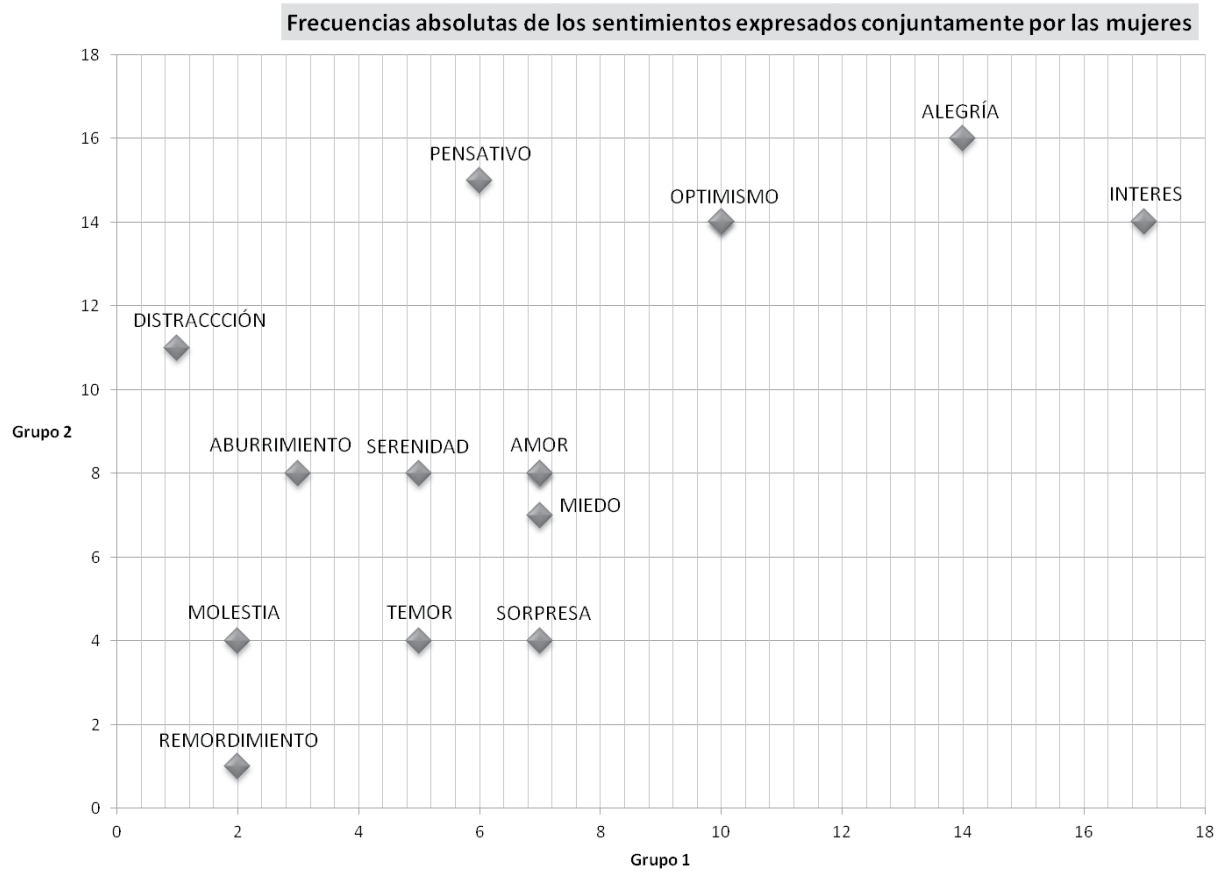

En la figura 6, se observa una gran dispersión entre las frecuencias de los sentimientos manifestados por ambos grupos de alumnas. Pero, a pesar de este hecho, se observó una convergencia alta en cuanto a su percepción sobre dichos sentimientos.

Por otra parte, en el caso de los alumnos, el coeficiente de correlación mostró una relación positiva media $(0.65)$ con respecto

a los puntajes obtenidos en los sentimientos expresados por cada uno de ellos (figura 7). También destacan las frecuencias obtenidas para la alegría y la confianza, ya que en ambos grupos fueron las mismas (es decir, las diferencias entre ambos grupos es 0 (figura 8), aunque los puntos en el diagrama de dispersión muestran un mayor aglutinamiento o concreción (figura 9). 
Figura No. 7. Frecuencias absolutas de ambos grupos de alumnos.

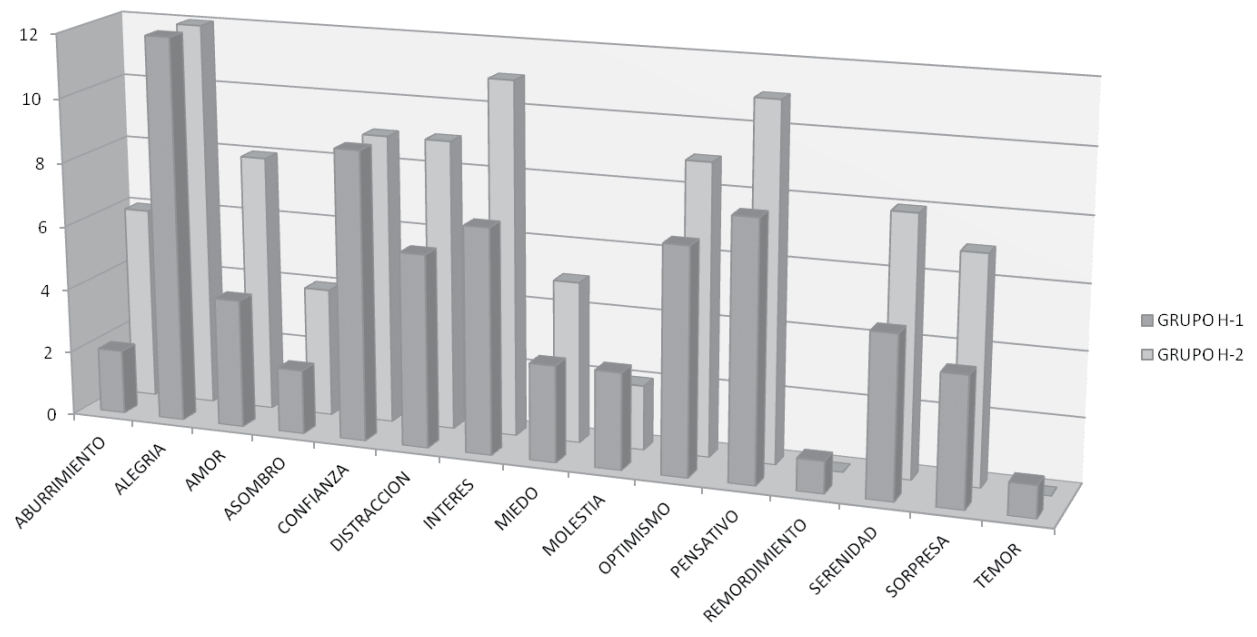

Fuente: Elaboración propia.

Figura No. 8. Oscilación observada en los sentimientos mencionados por ambos grupos de alumnos.

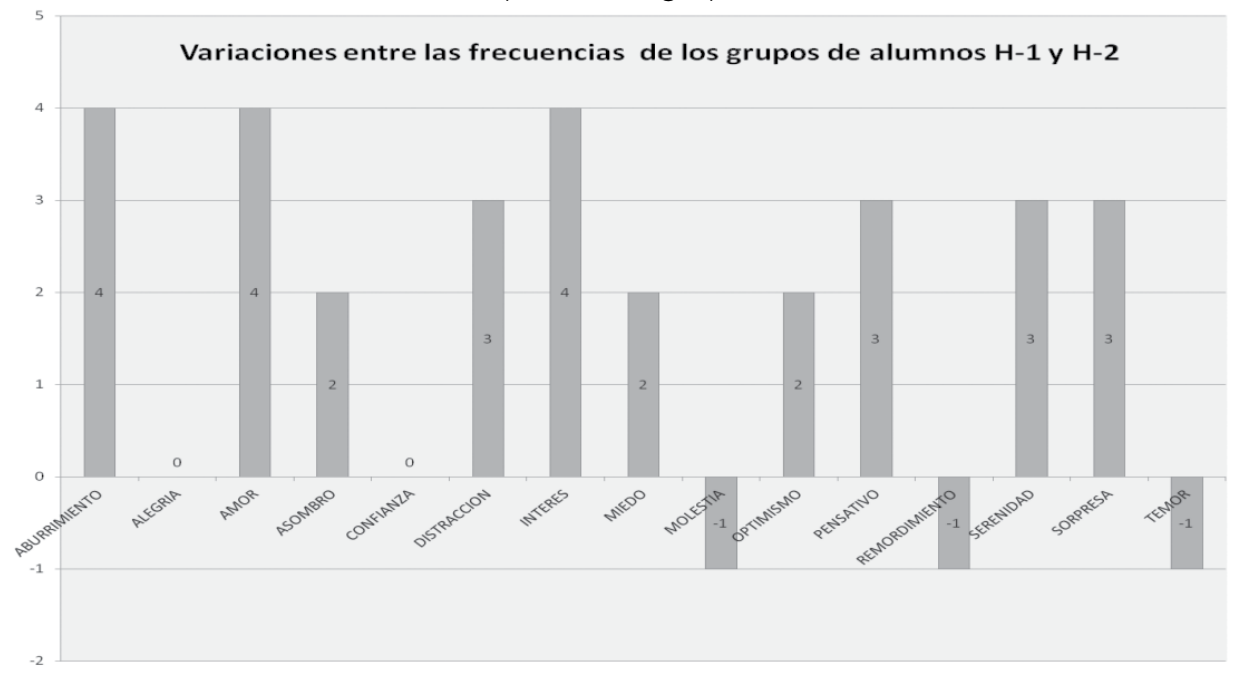

Fuente: Elaboración propia. 
Figura No. 9. Diagrama de dispersión de los sentimientos de ambos grupos de alumnos. .

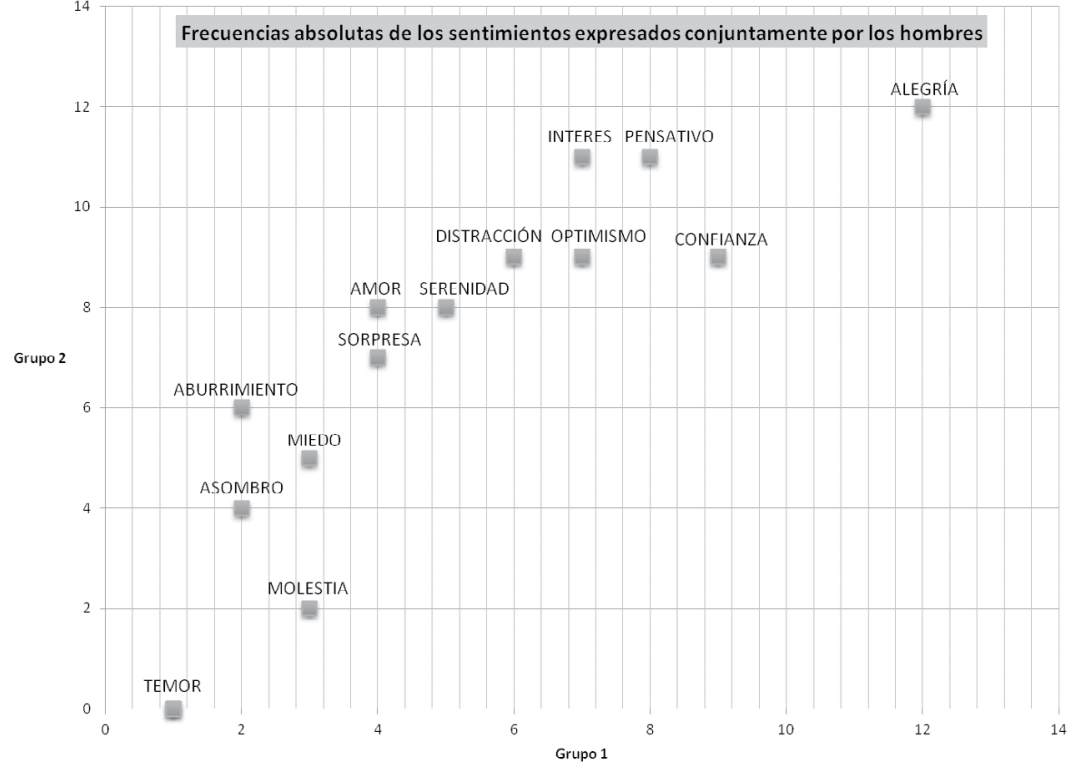

Fuente: Elaboración propia

Por último, si se analizan conjuntamente los datos de ambos grupos (mujeres y hombres) como si fuesen uno solo, de nuevo el coeficiente de correlación muestra una relación positiva fuerte $(0.86)$ con respecto a los puntajes obtenidos en cada uno de los sentimientos percibidos (figura 11).

Figura No. 10. Frecuencias absolutas de ambos grupos de alumnas y alumnos.

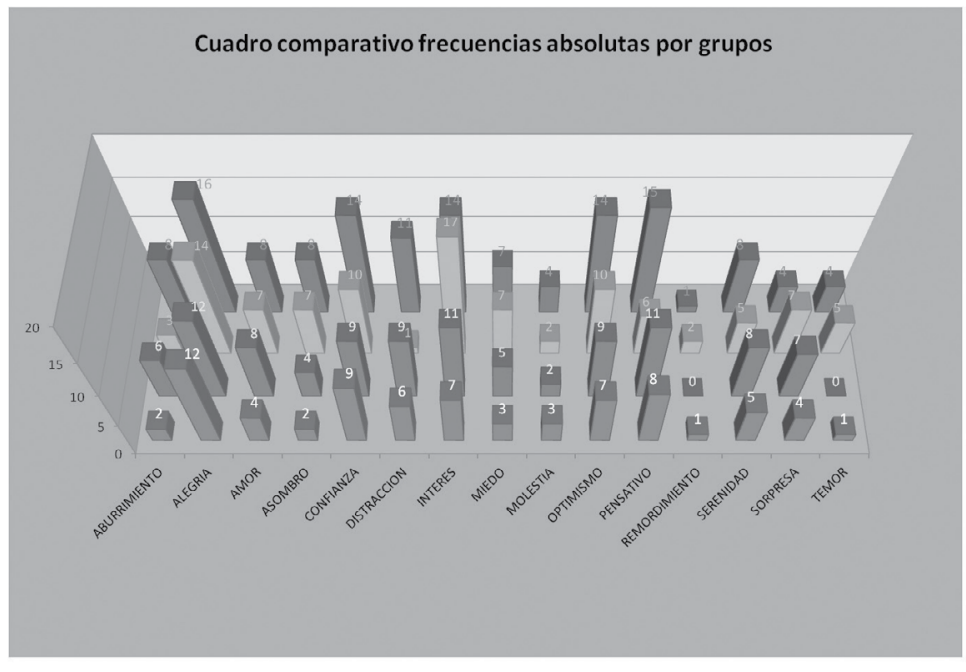

aGRUPOH-1 @GRUPOH-2 @GRUPOM-1 @GRUPOM-2

Fuente: Elaboración propia. 
Figura No. 11. Diagrama de dispersión de los sentimientos de ambos grupos de alumnas y alumnos.

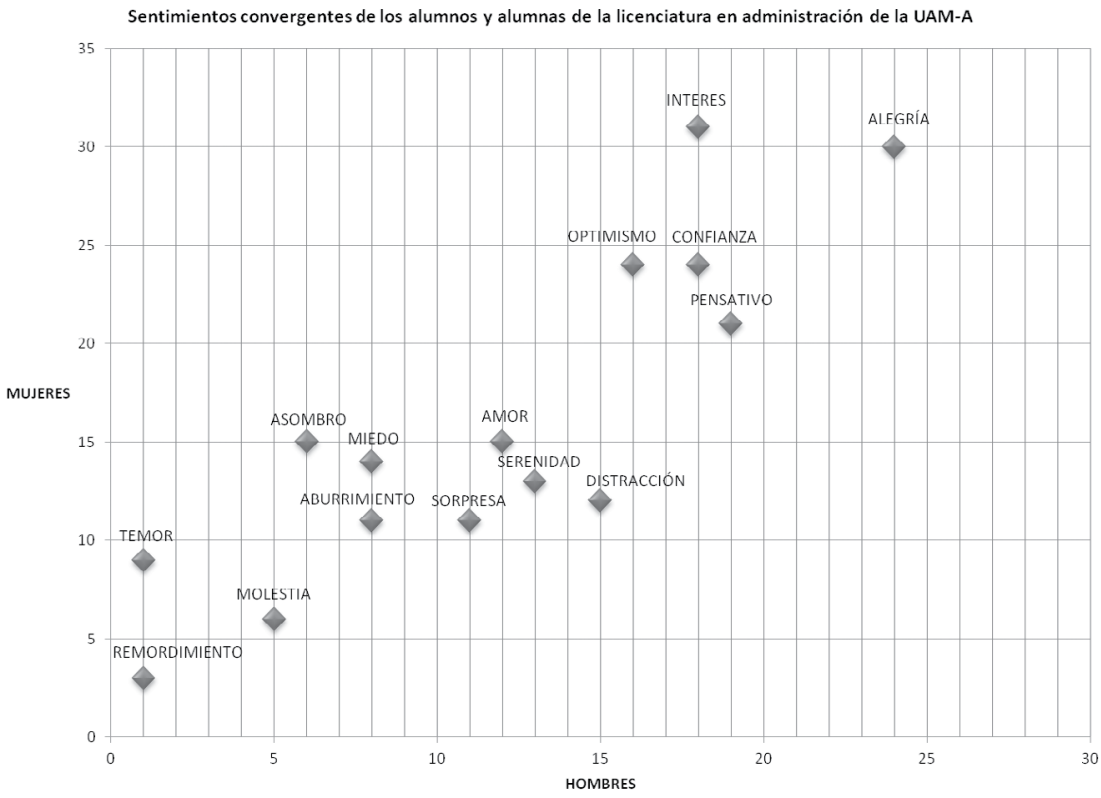

Fuente: Elaboración propia.

Los sentimientos con mayor frecuencia absoluta expresados por las alumnas y los alumnos fueron el interés, la alegría, el optimismo, la confianza y el estar pensativos. En un rango intermedio, se encuentran el asombro, el amor, el miedo, la serenidad, la distracción, el aburrimiento y la sorpresa. Los niveles más bajos los ocuparon el temor, la molestia y el remordimiento (figura 11). El desprecio y la agresividad obtuvieron valores bajos y tan solo fueron nombrados por las mujeres. Por esta razón se dejaron fuera de la figura.

A partir de la información anterior, se puede inferir la imagen proyectada (trabajo de cara) de los alumnos hacia sus compañeros y el resto de las personas con quienes tienen algún tipo de comunicación (interacción intercultural). Esta imagen es consistente con ser vistos como personas interesadas, alegres, optimistas, confiadas y reflexivas.
De manera simultánea minimizan en su comportamiento el temor, la molestia, el remordimiento, la agresividad y el desprecio. La convergencia de ambos grupos sobre estos sentimientos se demuestra con los coeficientes de correlación obtenidos.

Hasta aquí se han obtenido algunos hallazgos con respecto a cómo se observan, cómo quieren ser vistos los alumnos de Administración y qué se encontró con respecto a su visión una vez egresados. Para dilucidar a este respecto, se acudió al Sistema de Información de Egresados y Empleadores integrado por diversas encuestas realizadas por la UAM a sus egresados y potenciales empleadores.

Aquí se recatan algunos resultados de una encuesta aplicada a las generaciones 2005-2008. En ella se les preguntó por los distintos recursos utilizados para obtener su primer empleo y el actual. Destaca 
el uso de internet, la recomendación de algún familiar o amigo, bolsa de trabajo o externa, y lo más importante para los fines del presente trabajo: la recomendación de amigos de la licenciatura. La incidencia de este factor para obtener el primer empleo oscila entre 6 y $7 \%$. Si se aplicase este factor a las generaciones de egresados de los últimos cinco años, el resultado sería de entre 54 y 63 egresados, lo cual representa una tasa muy baja con respecto a otros medios de difusión. Con respecto al trabajo actual, este rubro no aumenta al mantenerse en $6 \%$, es decir, 54 egresados de las últimas generaciones acudirían a sus amigos de la Universidad por alguna recomendación o ayuda para contratarse. De igual manera se observa una baja incidencia de $3 \%$ en la generación 2005 y nula en la de 2008 al preguntarles sobre haber buscado la recomendación de algún profesor (figura 12).

Por otra parte, la mayoría de los egresados de la Licenciatura en Administración de las generaciones 2005-2008 se desempeñan en puestos de nivel intermedio en empresas consideradas de tamaño grande, como jefes de departamento, jefe de oficina/sección/ área, empleado profesional, ejecutivo de cuenta/venta y analista técnico. Además en ambas generaciones dijeron que si pudiesen se volverían a inscribir en la misma licenciatura (2005/75.9 \% y 2008/78.7\%). Y con respecto a la misma Universidad, la mayoría respondió de manera afirmativa (2005/93.1 \% y 2008/96.7\%).

Figura No. 12.

\begin{tabular}{|c|c|c|}
\hline & $\begin{array}{c}2005 \\
\%\end{array}$ & $\begin{array}{c}2008 \\
\%\end{array}$ \\
\hline Bolsa de trabajo de la UAM & 3.1 & 0.0 \\
\hline Bolsa de trabajo externa & 12.5 & 13.8 \\
\hline Anuncio de periodico & 9.4 & 0.0 \\
\hline Anuncio de una empresa o institucion & 0.0 & 0.0 \\
\hline Recomendacion de amigos de la licenciatura & 6.3 & 6.9 \\
\hline Recomendacion de un profesor & 3.1 & 0.0 \\
\hline Recomendacion de un amigo o familiar & 15.6 & 17.2 \\
\hline Relaciones hechas en empleos anteriores & 3.1 & 0.0 \\
\hline Creacion de un negocio, despacho, empresa propio & 3.1 & 6.9 \\
\hline Integracion a un negocio familiar & 0.0 & 0.0 \\
\hline Servicio social & 3.1 & 6.9 \\
\hline Practicas o estancias profesionales & 9.4 & 0.0 \\
\hline Agencia de colocacion & 6.3 & 13.8 \\
\hline Internet & 21.9 & 34.5 \\
\hline Difusion de curriculum & 0.0 & 0.0 \\
\hline Otro & 3.1 & 0.0 \\
\hline
\end{tabular}

\begin{tabular}{|c|c|c|}
\hline & 2005 & 2008 \\
\hline & $\%$ & $\%$ \\
\hline Bolsa de trabajo de la UAM & 3 & 3 \\
\hline Bolsa de trabajo externa & 6.1 & 12.1 \\
\hline Anuncio de periódico & $\mathrm{o}$ & 0 \\
\hline Anuncio de una empresa o institución & 0 & 0 \\
\hline Recomendación de amigos de la licenciatura & 6.1 & 6.1 \\
\hline Recomendación de un profesor & 3 & 0 \\
\hline Recomendación de un amigo o familiar & 18.1 & 15.2 \\
\hline Relaciones hechas en empleos anteriores & 9.1 & 3 \\
\hline Creación de un negocio, despacho, empresa propios & 12.1 & 6.1 \\
\hline Integración a un negocio familiar & 6.1 & 3 \\
\hline Servicio social & 6.1 & 3 \\
\hline Prácticas o estancias profesionales & 6.1 & 0 \\
\hline Agencia de colocación & 12.1 & 15.2 \\
\hline Internet & 12.1 & 27.2 \\
\hline Difusión de currículum & 0 & 0 \\
\hline Otro & 0 & 6.1 \\
\hline
\end{tabular}

Fuente: Azcapotzalco - Informes Descriptivos por División y Plan de Estudios. 


\section{A manera de conclusión}

Como resultado de los elementos de investigación aquí recabados, se pueden establecer las siguientes conjeturas. Los sentimientos son diferentes de las emociones. Los sentimientos poseen características distintas, como su origen, la duración y su participación en la construcción de la sociedad. Esto se realiza mediante el establecimiento de rituales y convenciones colectivas a partir de lo íntimo y lo privado, que elaboran un camino de doble vía a través de los andamiajes comunicativos entre los campos y los espacios sociales.

En suma, se pone en marcha la comunicación intercultural con un conjunto de actitudes cooperativas y disposiciones facilitadoras establecidas para compartir saberes, acciones, representaciones simbólicas y una pléyade de significados. La interculturalidad centra su éxito en el intercambio de significaciones y la resignificación de los supuestos intercambiados. Asimismo, opera bajo las reglas de interpretación implicadas en los intercambios de sentido gestados al calor de una situación comunicativa concreta, de ahí que sea bastante factible entender la comunicación intercultural como proceso comunicativo en el que se intercambian las representaciones del mundo, de uno mismo y de los otros, como espacios generadores de encuentros pero también de conflictos.

Por otra parte, los sentimientos definen la experiencia más destacada dentro de la vida afectiva de las personas. Para estudiar los fenómenos gestados por los sentimientos, se recurrió a la corriente del interaccionismo simbólico y en particular al microinteraccionismo individualista propuesto por el sociólogo canadiense Erving Goffman, porque sus conceptos permitieron descubrir algunos fenómenos presentes en el problema de investigación.

Este se centró en determinar los sentimientos involucrados en los alumnos adscritos a la Licenciatura en Administración de la UAM Azcapotzalco, con el objetivo expreso de saber cómo se identifican con la institución y con sus compañeros. En términos de Goffman, determinar su "trabajo de cara", su personaje, su desempeño como actores sociales, cómo quieren ser vistos y cómo informan a los demás de sus pretensiones y el grado esperado de cumplimiento de ellos para con el resto de las personas.

Los resultados obtenidos bosquejaron el "trabajo de cara" de los alumnos consistente en su deseo de ser vistos como alguien alegre, optimista, confiado, confiable e interesado en las actividades desarrolladas en su vida cotidiana. En suma, están definiendo su estatus social y los parámetros de sus interacciones comunicativas.

Por otra parte, y con respecto a la pregunta inicial del documento, se puede observar un nexo débil entre los egresados de la Licenciatura en Administración, debido al número tan reducido de egresados en busca de sus compañeros para una recomendación u oportunidad de empleo. Contrariamente a lo que opinan de su formación y de la Universidad donde estudiaron, ya que una gran mayoría volvería a estudiar en la UAM Azcapotzalco y adscritos a la Licenciatura en Administración.

\section{Referencias}

Álvarez Rodríguez, J. (2002). Análisis descriptivo de los valores sentimiento y emoción en la formación de profesores de la Universidad de Granada. Profesorado. Revista de Curriculum y Formación del Profesorado, 
6(1-2). Recuperado de http://digibug.ugr.es/ bitstream/10481/14995/1/rev61COL8.pdf Arndt, P. A. (2012). Design of learning spaces: Emotional and cognitive effects of learning environments in relation to child development. Mind, Brain, and Education, 6(1), 41-48.

Bordieu, P. (1990). Sociología y cultura. México: Grijalbo.

Caballero, J. J. (1998). La interacción social en Goffman. Reis: Revista Española de Investigaciones Sociológicas, 83, 121-149.

Capdevielle, J. (2011). El concepto de habitus: "con Bordieu y contra Bordieu". Anduli: Revista Andaluza de Ciencias Sociales, 10, 31-46.

Ciompi, L. (2007). Sentimientos, afectos y lógica afectiva: su lugar en nuestra comprensión del otro y del mundo. Revista de la Asociación Española de Neuropsiquiatría, 27(2), 153-171.

Collins, R. (2014). Interaction ritual chains. Princeton, Oxford: Princeton University Press.

Consier, J. (2015). Psychologie des émotions et des sentiments. Recuperado de http:// icar.univ-lyon2.fr/membres/jcosnier/Emotions_et_sentiments.pdf

El cerebro, el universo dentro de nosotros: percepción (s/f). Recuperado de https:// www.youtube.com/watch?v=Q0vjD0NI9uk

Escudero, J. (2007). Hacia una fenomenología de los afectos: Martin Heidegger y Max Scheler. THEMATA. Revista de Filosofia, 39, 365-68.

Foucault, M. (2009). La hermenéutica del sujeto. México: Fondo de Cultura Económica.

Gabás Pallás, R. (2002). La fenomenología de los sentimientos en Max Scheler y el concepto de afección en Martin Heidegger. Enrahonar: quaderns de filosofia, 34, 31-46.
García, M. R. (2011). De personas, rituales y máscaras: Erving Goffman y sus aportes a la comunicación interpersonal. Quórum Académico, 8(15), 78-94.

Gaytán, G. (2011). Los sentimientos según Max Scheler. Recuperado de http://rmgpmotivacion.blogspot.com.co/2011/11/lossentimientos-segun-max-scheler.html

Goffman, E. (1951). Symbols of class status. The British Journal of Sociology, 2(4), 294-304.

Goffman, E. (1956). The nature of deference and demeanor. American Anthropologist, 58(3), 473-502.

Goffman, E. (1970). Ritual de la interacción. Buenos Aires, Ed. Tiempo Contemporáneo. Goffman, E. (1990). The presentation of self in everyday life. Londres: Penguin Books.

González-Arratia, N. I., Valdez Medina, J. L. y Serrano García, J. M. (2015). Autoestima en jóvenes universitarios. CIENCIA ergo-sum, 10(2), 173-179.

Guerra Manzo, E. (2010). Las teorías sociológicas de Pierre Bourdieu y Norbert Elias: los conceptos de campo social y habitus. Estudios Sociológicos, 28(83), 383-409

Heller, A. (1999), Teoría de los sentimientos, Barcelona, Ediciones Coyoacán.

Hentschel, K. (2001). Moleküle der Gefühle,, Neurobiologische Grundlagen der Sucht. Recuperado de http://www.koerper-psychetherapie.ch/media/Dokumente/Molekuele $\% 20$ der $\% 20$ Gefuehle $\% 20$ Gesamt $\% 20$ Text.pdf

Herrera Gómez, M. y Soriano Miras, R. M. (2004). La teoría de la acción social en Erving Goffman. Papers: revista de sociología, 73, 59-79.

Hochschild, A. (2008). La mercantilización de la vida intima: apuntes de la casa y el trabajo. Buenos Aires: Katz. 
Hormigos Ruiz, J. y Oda Ángel, F. (2014). Identidades culturales en un mundo en movimiento: reflexiones desde la sociología. methaodos. revista de ciencias sociales, 2(2), 244-255.

Iranzo, J. M. (2005). Reseña de "Limits to Growth: The 30-Year Update" de Meadows, Donella; Randers, Jorgen y Meadows, Dennis. EMPIRIA. Revista de Metodología de las Ciencias Sociales, 10, 23-235.

Javeau, C. (2010). Sartre et sa théorie des émotions: une confrontation avec Erving Goffman. SociologieS, Files, Émotions et sentiments, réalité et fiction. Recuperado de http://sociologies.revues.org/3169

Laneri, N. (ed.) (2007). Performing death: Social analyses of funerary traditions in the ancient Near East and Mediterranean. Chicago: The Oriental Institute.

La Recherche (2000). Entrevista con Pierre Bordieu: la sociología ¿es una ciencia? Recuperado de http://www.antroposmoderno. com/antro-articulo.php?id_articulo= 123

López Lara, Á. F. y Reyes Ramos, M. E. (2010). Erving Goffman: microinteracción y espacio social. Veredas. Revista del Pensamiento Sociológico, 115-136.

Luborsky, M. R. y Rubinstein, R. L. (2011). Sampling in qualitative research rationale, issues, and methods. En A. Bryman, Ethnography (t. II, pp. 122-140). Londres: Sage Publications.

Manzo, E. G. (2010). Las teorías sociológicas de Pierre Bourdieu y Norbert Elias: los conceptos de campo social y habitus. Estudios Sociológicos, 28(83), 383-409.

Masoliver Ródenas, J. A. (1989). La Quincena Soviética, de Vicente Molina Foix: entre el juego y la farsa. Insula: Revista de Letras $y$ Ciencias Humanas, 509, 21-22.

Martínez Samos, A. (2014). "La quincena soviética” (1988) de Vicente Molina Foix: del examen de la identidad a la imposibilidad del amor. Pasavento: Revista de Estudios Hispánicos, 2(1), 207-224.

Mercado Maldonado, A. y Zaragoza Contreras, L. (2011). La interacción social en el pensamiento sociológico de Erving Goffman. Espacios Públicos, 14(31), 158-175.

Nieves Achón, Z., Satchimo Namalyongo, A., González Nieves, Y., Jiménez Jorge, M. (2013). Algunas consideraciones acerca del estrés académico en los estudiantes universitarios. Revista Psicoespacios, 7(11), 91-116. Recuperado de http://revistas.iue. edu.co/index.php/Psicoespacios/article/ view/215/621

Otero, M. R. (2006). Emociones, sentimientos y razonamientos en didáctica de las ciencias. Revista Electrónica de Investigación en Educación en Ciencias, 1(1). Recuperado de http://ppct.caicyt.gov.ar/index.php/reiec/ article/view/7350

Pech Salvador, C., Rizo García, M. y Romeu Aldaya, V. (2009). El habitus y la intersubjetividad como conceptos clave para la comprensión de las fronteras internas: un acercamiento desde las propuestas teóricas de Bourdieu y Schütz. Frontera Norte, 21(41), 33-52.

Pulido Acosta, F. y Herrera Clavero, F. (2016). Miedo y rendimiento académico universitario en el contexto pluricultural de Ceuta. Revista PsicologíaCientifica.com. Recuperado de http://www.psicologiacientifica. com/miedo-rendimiento-academico-universitario-ceuta/

Rizo García, M. (s/f). La dimensión emocional de la interacción. Lecturas desde la propuesta microsociológica de Randall Collins. Recuperado de http://amic2014. uaslp.mx/g15/g15_07.pdf 
Scherer, K. R. (2005). What are emotions? And how can they be measured? Social Science Information, 44(4), 695-729.

Scheler, M. (1992). On feeling, knowing, and valuing: Selected writings. Chichago y Londres: University of Chicago Press.

Quinn, M. (2001). Purposeful sampling. En A. Bryman (ed.), Ethnography (t. II, pp. 106-121). Londres: Sage.

Universidad Autónoma Metropolitana (s/f). Azcapotzalco: Resultados del estudio de seguimiento de egresados generacionales 20052008. Recuperado de http://www.uam.mx/ egresados/r_d/azc/index.php 